\title{
BMJ Open Efficacy of iron supplementation on fatigue and physical capacity in non- anaemic iron-deficient adults: a systematic review of randomised controlled trials
}

\author{
Brett L Houston, ${ }^{1,2}$ Daryl Hurrie, ${ }^{3}$ Jeff Graham, ${ }^{1,2}$ Brittany Perija, ${ }^{4}$ Emily Rimmer, ${ }^{1,2}$ \\ Rasheda Rabbani, ${ }^{5,6}$ Charles N Bernstein, ${ }^{7}$ Alexis F Turgeon, ${ }^{8,9}$ \\ Dean A Fergusson, ${ }^{10}$ Donald S Houston, ${ }^{1,2}$ Ahmed M Abou-Setta, ${ }^{5,6}$ \\ Ryan Zarychanski ${ }^{1,2,5,6}$
}

To cite: Houston BL, Hurrie D, Graham J, et al. Efficacy of iron supplementation on fatigue and physical capacity in non-anaemic irondeficient adults: a systematic review of randomised controlled trials. BMJ Open 2018;8:e019240. doi:10.1136/ bmjopen-2017-019240

- Prepublication history and additional material for this paper are available online. To view these files, please visit the journal online ( http://dx.doi. org/10.1136/bmjopen-2017019240).

Received 11 September 2017 Revised 1 February 2018 Accepted 12 February 2018

Check for updates

For numbered affiliations see end of article.

\section{Correspondence to}

Dr Ryan Zarychanski;

rzarychanski@cancercare.

mb.ca

\section{ABSTRACT}

Objective Iron supplementation in iron-deficiency anaemia is standard practice, but the benefits of iron supplementation in iron-deficient non-anaemic (IDNA) individuals remains controversial. Our objective is to identify the effects of iron therapy on fatigue and physical capacity in IDNA adults.

Design Systematic review and meta-analysis of randomised controlled trials (RCTs).

Setting Primary care.

Participants Adults ( $\geq 18$ years) who were iron deficient but non-anaemic.

Interventions Oral, intramuscular or intravenous iron supplementation; all therapy doses, frequencies and durations were included.

Comparators Placebo or active therapy.

Results We identified RCTs in Medline, Embase, Cochrane Central Register of Controlled Trials, Cumulative Index of Nursing and Allied Health, SportDiscus and CAB Abstracts from inception to 31 October 2016. We searched the WHO's International Clinical Trials Registry Platform for relevant ongoing trials and performed forward searches of included trials and relevant reviews in Web of Science. We assessed internal validity of included trials using the Cochrane Risk of Bias tool and the external validity using the Grading of Recommendations Assessment, Development and Evaluation methodology. From 11580 citations, we included 18 unique trials and 2 companion papers enrolling 1170 patients. Using a Mantel-Haenszel random-effects model, iron supplementation was associated with reduced selfreported fatigue (standardised mean difference (SMD) $-0.38 ; 95 \% \mathrm{Cl}-0.52$ to $-0.23 ; I^{2}$ 0\%; 4 trials; 714 participants) but was not associated with differences in objective measures of physical capacity, including maximal oxygen consumption (SMD $0.11 ; 95 \% \mathrm{Cl}-0.15$ to 0.37 ; $\mathrm{I}^{2} 0 \%$; 9 trials; 235 participants) and timed methods of exercise testing. Iron supplementation significantly increased serum haemoglobin concentration (MD $4.01 \mathrm{~g} / \mathrm{L}$; $95 \% \mathrm{Cl} 1.22$ to $6.81 ; I^{2} 48 \%$; 12 trials; 298 participants) and serum ferritin (MD $9.23 \mu \mathrm{mol} / \mathrm{L} ; 95 \% \mathrm{Cl} 6.48$ to 11.97 ; $\mathrm{I}^{2} 58 \%$; 14 trials; 616 participants).

\section{Strengths and limitations of this study}

- We used a comprehensive search strategy, an a priori protocol and adhered to established methodological (eg, Preferred Reporting Items for Systematic Reviews and Meta-Analyses, Grading of Recommendations Assessment, Development and Evaluation) guidelines.

- We identified an at-risk patient population, for whom iron deficiency is highly prevalent, but treatment is unknown.

- Our outcomes are clinically relevant and patient centred.

- Our search was limited to English studies.

- In an effort to quantify elemental iron administration, we did not include studies evaluating dietary iron fortification.

Conclusion In IDNA adults, iron supplementation is associated with reduced subjective measures of fatigue but not with objective improvements in physical capacity. Given the global prevalence of both iron deficiency and fatigue, patients and practitioners could consider consumption of iron-rich foods or iron supplementation to improve symptoms of fatigue in the absence of documented anaemia.

PROSPERO registration number CRD42014007085.

\section{INTRODUCTION}

Iron deficiency (ID) is estimated to affect two billion people and is the leading cause of anaemia worldwide. ${ }^{12}$ Iron is necessary for cellular immune responses, oxidative metabolism within the mitochondria and production of haemoglobin and myoglobin. When iron losses exceed dietary iron absorption, iron stores become depleted resulting in impaired haemoglobin production and decreased red 
blood cell haemoglobin content. ${ }^{3}$ Reduction in haemoglobin concentration below a threshold (conventionally defined by the WHO as $120 \mathrm{~g} / \mathrm{L}$ for women and $130 \mathrm{~g} / \mathrm{L}$ for men) signifies anaemia. ${ }^{4}$

It is well established that anaemia results in decreased physical capacity and increased fatigue proportional to anaemia severity. ${ }^{5-9}$ Unfortunately, patient-reported fatigue is common in community and primary care settings with a prevalence ranging from $7 \%$ to $45 \% .{ }^{10}$ It is estimated that the indirect annual economic consequence of chronic fatigue in the USA is 9.1 billion dollars. ${ }^{11}$

The clinical relevance of iron deficiency in the absence of anaemia is poorly understood but may impact wellbeing, perceptions of fatigue or contribute to decrements in physical performance through impairment in biochemical processes including tissue and mitochondrial oxidative capacity. ${ }^{8}$ While iron replacement can normalise haemoglobin concentration, restore work capacity and improve fatigue in iron-deficiency anaemia, it is unclear if supplementation affects fatigue and physical capacity in iron-deficient but non-anaemic (IDNA) individuals. In the absence of compelling efficacy data on well-being or muscle function, the use of iron supplements is common in the general population and are routinely recommended to high performance athletes to enhance performance.

Given the global prevalence of iron deficiency and impact of fatigue, the purpose of this systematic review is to identify, critically appraise and meta-analyse data from prospective randomised trials evaluating iron therapy in adults with IDNA.

\section{METHODS}

Using an a priori published protocol (CRD42014007085; available at https://www.crd.york.ac.uk/PROSPERO/ $),{ }^{12}$ we conducted a systematic review using methodological approaches outlined in the Cochrane Handbook for Systematic Reviewers and reported according to the Preferred Reporting Items for Systematic Reviews and Meta-Analysis criteria. ${ }^{12-14}$ A panel of experts from multiple fields (eg, internal medicine, haematology, kinesiology, gastroenterology, research methodology) formulated the research question, reviewed search strategies and methods and provided input throughout the review process.

\section{Populations, interventions, comparators, outcome measures, setting and study designs}

Our research question was 'In iron-depleted but non-anaemic adults, does iron supplementation improve fatigue and physical capacity?' We included randomised controlled trials (RCTs) of adults ( $\geq 18$ years) who were iron deficient but non-anaemic (see online supplementary appendix 1). Interventions included oral, intramuscular or intravenous iron supplementation; all therapy doses, frequencies and durations were included. We included trials that evaluated outcomes at least 28 days from the initiation of iron therapy. Comparators included placebo or active therapy. Our exclusion criteria are presented in online supplementary appendix 2.

Our primary outcome measures were self-reported fatigue and objective measures of physical capacity. Secondary outcomes included the incidence of anaemia, change in haemoglobin concentration and serum ferritin and the incidence of adverse outcomes including iron toxicity, constipation, diarrhoea, gastrointestinal intolerance and nausea.

\section{Search strategy for identification of studies}

We searched Medline, Embase, Cochrane Central Register of Controlled Trials, Cumulative Index of Nursing and Allied Health, SportDiscus and CAB Abstracts from inception to 31 October 2016 to identify relevant citations of published trials, using individualised systematic search strategies for each database. The Medline strategy is presented in online supplementary appendix 3. We searched the WHO's International Clinical Trials Registry Platform, clinicaltrials.gov and relevant conference proceedings to identify planned, ongoing or recently completed but unpublished trials. We performed forward searches of included trials and relevant reviews in Web of Science to identify additional citations and contacted study authors to request pertinent unpublished data or provide clarifications on study methods or results. Reference lists of narrative and systematic reviews and of the included trials were searched for additional citations. We performed reference management in EndNote V.X7 (Thomson Reuters).

\section{Study selection, data extraction and quality assessment}

We screened citations, selected studies and extracted data from included trials using standardised and piloted screening and data extraction forms. Citation screening, study selection and data extraction were performed in duplicate. The following data were extracted from each trial: author identification, publication year, publication language, trial location, source of trial funding, participant characteristics (age, sex, weight), intervention/ comparator (drug used, dose (elemental iron), route of administration, duration), as well as results for the primary and secondary outcomes. We assessed the internal validity of included trials using the Cochrane Collaboration Risk of Bias tool. ${ }^{13}$ Discrepancies between the two reviewers were resolved by consensus or by a third reviewer (RZ), as required. Data extraction and descriptive statistics were performed using Microsoft Excel 2016 V.15.

\section{Data analysis}

Data analysis was performed using Review Manager (RevMan V.5.3.5, The Nordic Cochrane Centre, The Cochrane Collaboration). Study level comparisons of dichotomous data were presented as risk ratios (RR) with 95\% CI. Pooled continuous data were expressed as the mean difference (MD) or standardised mean difference (SMD). Change scores or post-treatment means were extracted to inform summary estimates for continuous 
data. Pooled RRs and 95\% CIs were calculated using Mantel-Haenszel random-effects model. Pooled MDs or SMDs were calculated using a random-effects model. For the primary outcome of fatigue, if multiple scales were reported, fatigue-specific scores were preferred over general scores, and the most commonly reported and clinically meaningful score was used to generate summary effect measures. In studies evaluating exercise capacity, weight-based maximal oxygen consumption $\left(\mathrm{VO}_{2} \max \right)$ values were used preferentially if both absolute and weight-based $\mathrm{VO}_{2}$ max results were provided. Statistical heterogeneity was quantified using the $\mathrm{I}^{2}$ statistic. ${ }^{15}$ For the primary outcomes of fatigue and work capacity, we evaluated potential publication bias using funnel plot analysis. ${ }^{16}$ All tests of statistical inference reflect a two-sided alpha of 0.05 .

\section{Subgroup analyses}

We performed subgroup analyses for fatigue and exercise capacity outcomes according to biological sex, athletic status (athlete or non-athlete), method of iron administration, duration of therapy, duration of study follow-up and risk of bias.

\section{Grading the evidence}

We graded the strength of evidence for our primary outcomes using the Grading of Recommendations Assessment, Development and Evaluation methodology. This approach classifies the strength of evidence as 'high', 'moderate', 'low' or 'very low.'

\section{RESULTS}

\section{Trial characteristics and study populations}

Of the 11580 citations identified, we included 18 unique trials and 2 companion papers, ${ }^{1718}$ enrolling 1170 subjects (figure 1; table 1). Trials were published between 1989 and 2015, and all trials were published in peer-reviewed journals. Eight trials ${ }^{19-26}$ were from North America, seven trials $^{27-33}$ were from Europe, two trials ${ }^{18}$ were from Australia and one trial ${ }^{35}$ was from Asia.

Exclusively healthy women (aged 17 to 55 years old) with varying levels of fitness (sedentary to well trained) were enrolled in all but three studies. ${ }^{22} 2729$ The WHO cut-off for anaemia (haemoglobin concentration $\geq 130 \mathrm{~g} / \mathrm{L}$ (males) and $\geq 120 \mathrm{~g} / \mathrm{L}$ (females)) was used by nine studies, ${ }^{19}$ 22-25 30-32 35 whereas seven studies used lower values ranging from $\geq 110$ to $<120 \mathrm{~g} / \mathrm{L},{ }^{20} 212728333436$ and baseline haemoglobin concentration was not provided in two trial reports. ${ }^{26} 29$

All trials were placebo controlled. In 13 of 18 trials $(72 \%)$, we considered the blinding of participants and personnel to be adequate. Likewise, 10 trials (55\%) adequately incorporated blinded outcome assessment. One trial ${ }^{29}$ was considered to have a low risk of bias (table 2). The remainder of the trials were considered unclear risk of bias due to unclear processes of randomisation (12 trials $^{19-27} 303334$ ) or allocation concealment (13 trials $\left.{ }^{19-27} 30313435\right)$.

\section{Interventions}

Iron interventions consisted of iron supplementation administered orally, intramuscularly or intravenously. Of the trials evaluating oral supplements, all but one ${ }^{29}$ used ferrous sulfate (13 trials, ${ }^{19-263032333536} 713$ participants). Intravenous iron was administered in three trials ${ }^{27} 2831$ (395 participants), and intramuscular iron in one trial ${ }^{34}$ (16 participants). In trials using oral iron, ${ }^{19-26} 293032333536$ the mean daily elemental iron dose was $86.9 \mathrm{mg}( \pm 49.1 \mathrm{mg}$; range: $16-200 \mathrm{mg}$ ). In trials reporting intravenous iron, ${ }^{27} 2831$ the mean daily elemental iron dose was $566 \mathrm{mg}$ ( $\pm 330 \mathrm{mg}$; range $200-1000 \mathrm{mg}$ ) and mean total elemental iron dose $767 \mathrm{mg}$ ( $\pm 206 \mathrm{mg}$; range $500-1000 \mathrm{mg}$ ). Among all studies, the mean duration of iron therapy was 46 days ( \pm 30 days; range 1-112 days), and mean duration of follow-up was 57 days ( \pm 24 days; range 28-112 days).

\section{Primary outcomes}

Fatigue

Four trials ${ }^{28} 31-33$ enrolling 714 participants were eligible for meta-analysis. Iron supplementation was associated with a reduction in subjective measures of fatigue when assessed by either the Piper Fatigue Scale, ${ }^{28}$ the Current and Past Psychological State scale, ${ }^{32}$ visual analogue scale $^{33}$ or Brief Fatigue Inventory questionnaire (BFI) ${ }^{31}$ (SMD $-0.38 ; 95 \%$ CI -0.52 to $-0.23 ; \mathrm{I}^{2} 0 \%$ ) (figure 2 ). In one trial using the BFI score, fatigue was not significantly different between groups after 12 weeks, although it was improved in the subgroup of participants with the lowest iron stores (ferritin $\leq 15 \mathrm{ng} / \mathrm{mL}$ or transferrin saturation $\leq 20 \%) .{ }^{31}$ Evaluation of publication bias was not possible due to the low number of included trials. Given that the majority of trials were of unclear risk of bias, we graded the overall strength of evidence as moderate.

\section{Physical capacity}

Physical capacity was reported in 10 trials $^{19} 2022-273034$ (291 participants); all but one ${ }^{20}$ of the trials employed at least one of three common aerobic tests of physical capacity: time trial, ${ }^{22} 25$ time to exhaustion ${ }^{23} 262734$ or $\mathrm{VO}_{2} \max ^{192-273034}$ performance from a graded exercise test. In two trials (79 participants) that used $15 \mathrm{~km}$ time trials, ${ }^{22}{ }^{25}$ iron supplementation was not associated with improved exercise capacity (SMD $-0.09 ; 95 \%$ CI -0.53 to $0.35 ; \mathrm{I}^{2} 0 \%$ ) (figure 3A). In four trials ${ }^{23262734}$ (69 participants) that used time-to-exhaustion tests, iron supplementation did not significantly improve physical capacity (SMD $0.25 ; 95 \%$ CI -0.22 to $0.73 ; \mathrm{I}^{2} 0 \%$ ) (figure $3 \mathrm{~B}$ ). Nine trials ${ }^{1922-27} 3034$ (235 participants) reported $\mathrm{VO}_{2}$ max as a surrogate measure of physical capacity. Iron supplementation did not increase $\mathrm{VO}_{2}$ max (SMD 0.11; $95 \%$ CI -0.15 to $0.37 ; \mathrm{I}^{2} 0 \%$ ) (figure $3 \mathrm{C}$ ). We found no evidence of funnel plot asymmetry to suggest publication bias for this outcome (see online supplementary appendix 4). The overall strength of the evidence for time trial, time 


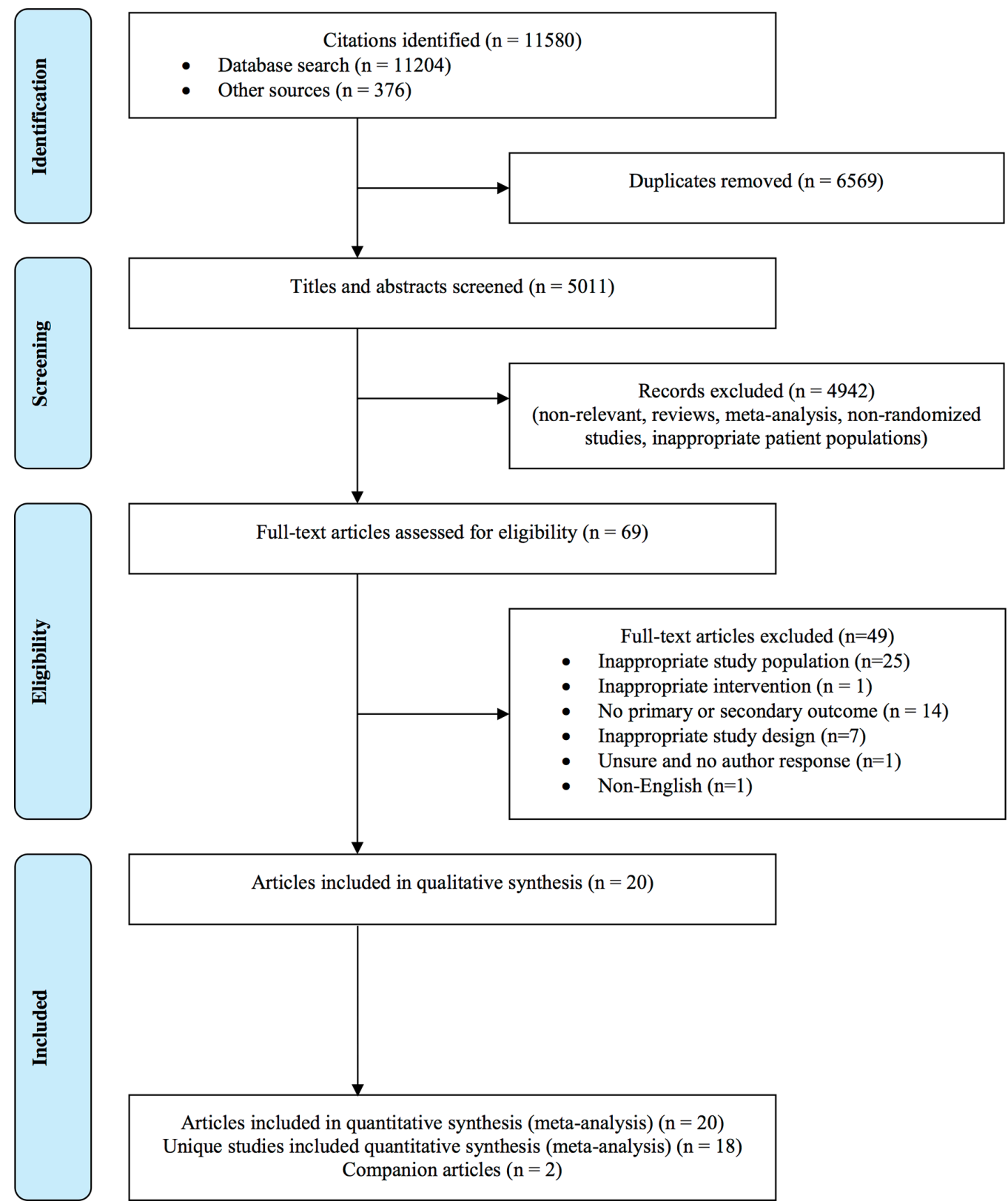

Figure 1 Study flow diagram following the Preferred Reporting Items for Systematic Reviews and Meta-Analyses ${ }^{14}$ with modifications. Of the 11580 citations identified, we included 18 unique trials and 2 companion papers.

to exhaustion and $\mathrm{VO}_{2}$ max outcomes were low, given the imprecision of effect estimates and that the majority of trials were of unclear risk of bias.

One trial ${ }^{20}$ (20 participants) used dynamic knee extension exercise to evaluate changes in physical capacity. In this trial, the decline in maximum voluntary contraction after 6 min of exercise was significantly less in participants randomised to receive iron. Among 16 other unique measures of physical capacity, 19\% (3 of 16) found statistically significant increases in measures of physical capacity with iron supplementation (see online supplementary appendix 5).

\section{Subgroup analysis}

Subgroup analyses based on method of iron administration and duration of follow-up demonstrated no statistically significant differences in subjective fatigue (see online supplementary appendix 6). Biological sex, athletic status (athlete vs non-athlete) and risk of bias could not be evaluated as all trials contributing data to the meta-analyses enrolled women of uncharacterised athletic status and were of unclear risk of bias. ${ }^{28} 31-33$ Subgroup analyses evaluating athletic status and method of iron administration demonstrated no statistically significant differences in objective physical capacity (see online supplementary appendix 7 ). Biological sex, duration of follow-up and risk of bias were unevaluable as all trials enrolled women with follow-up of less than 2 months and all were of unclear risk of bias. ${ }^{1922-27} 3034$

\section{Secondary outcomes and adverse events}

Despite the absence of baseline anaemia, iron supplementation significantly increased serum haemoglobin 


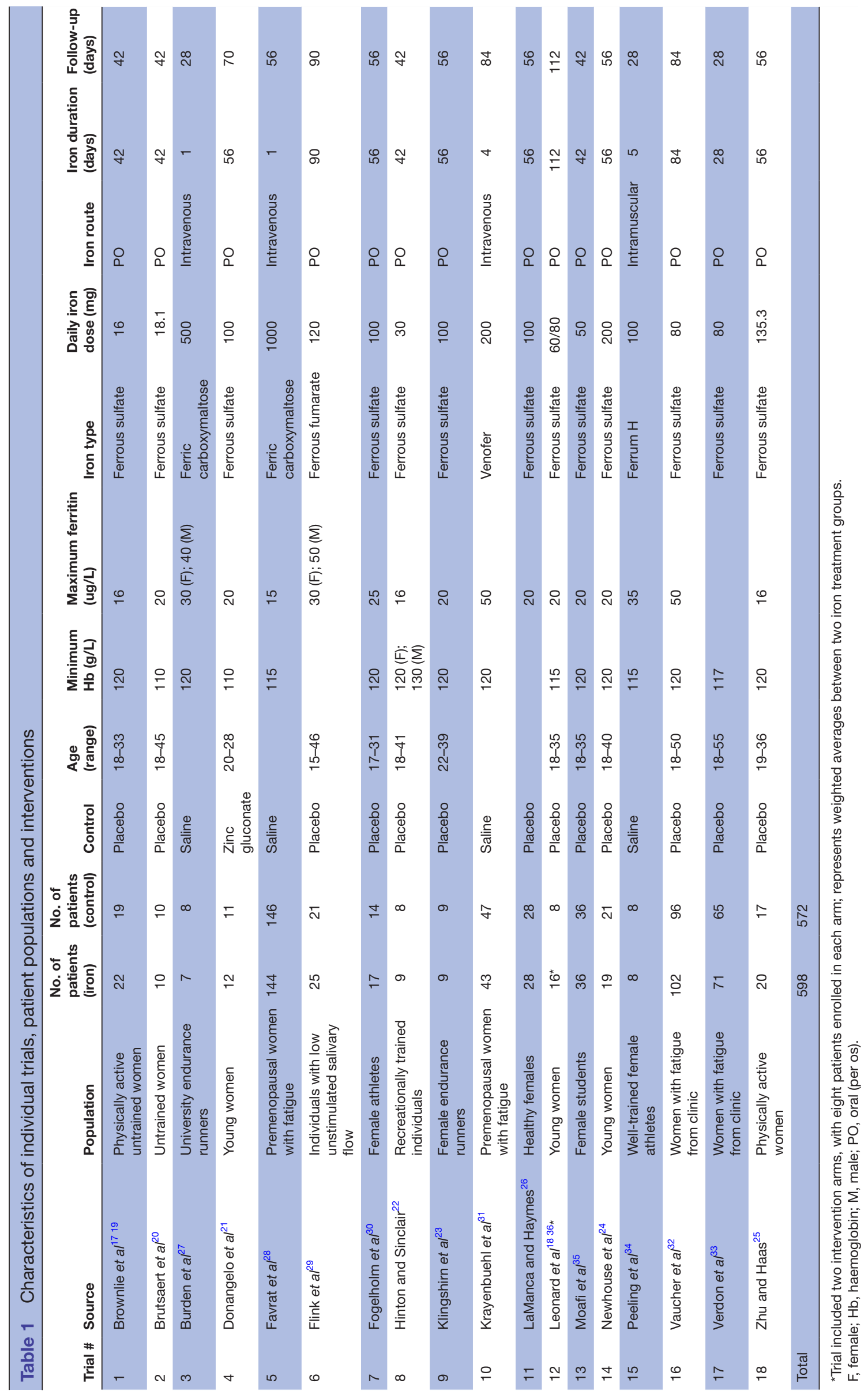


Table 2 Cochrane risk of bias summary

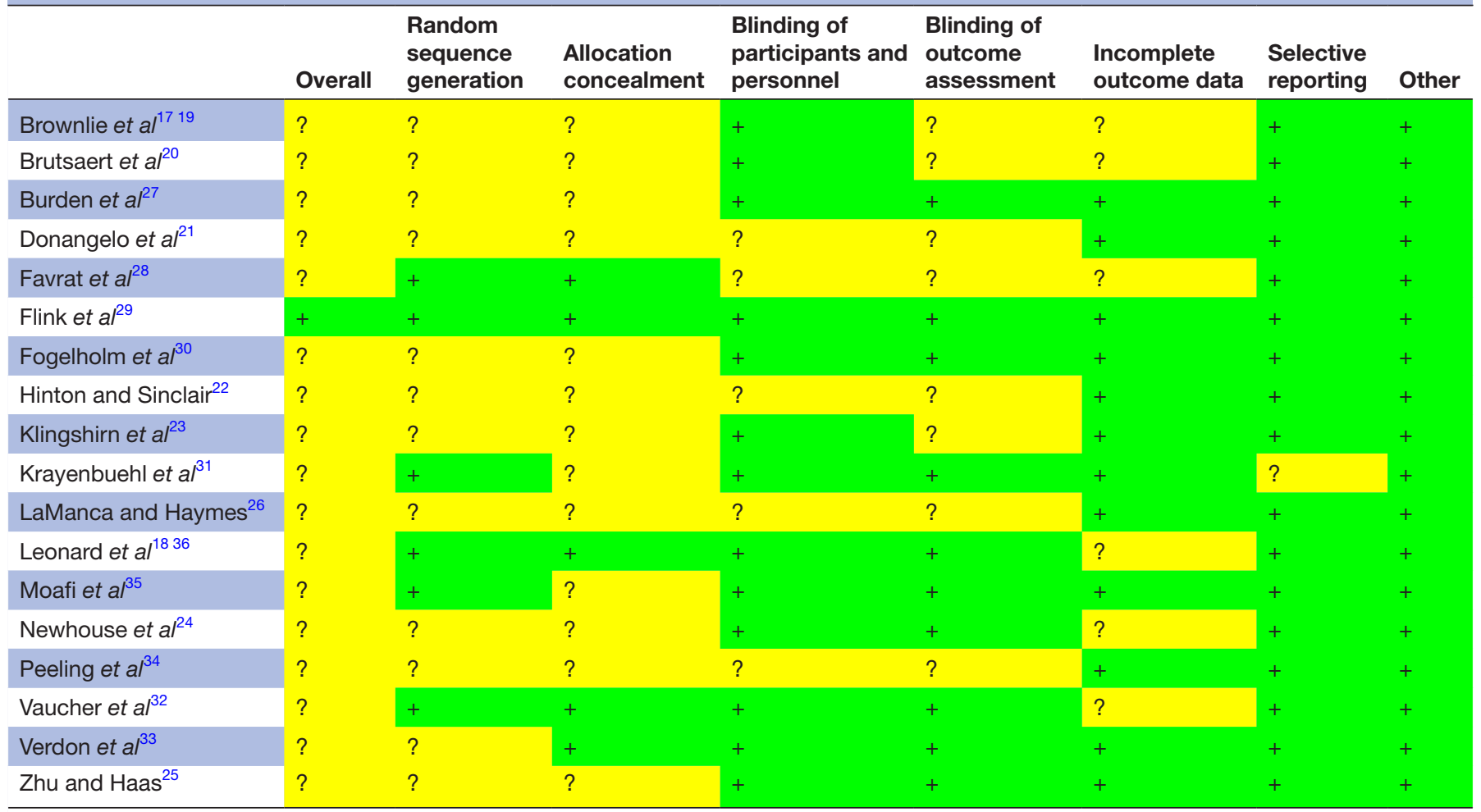

Green $(+)=$ low risk of bias; Yellow (?)=unclear risk of bias.

concentration (MD $4.01 \mathrm{~g} / \mathrm{L}$; $95 \%$ CI 1.22 to 6.81 ; I ${ }^{2} 48 \%$; 12 trials; 298 participants) ${ }^{18-27} 3034$ (see online supplementary appendix 8). In two trials, ${ }^{25} 28$ reporting incident anaemia, a new diagnosis of anaemia at trial completion was less common in patients randomised to receive iron supplementation. Iron supplementation also significantly increased serum ferritin (MD $9.23 \mu \mathrm{mol} / \mathrm{L} ; 95 \%$ CI 6.48 to 11.97 ; $\mathrm{I}^{2} 58 \%$; 14 trials; 616 participants) (see online supplementary appendix 9 ).

Adverse events were sparsely reported. Gastrointestinal intolerance was reported in three trials ${ }^{2329} 32$ and was significantly increased in one trial ${ }^{29}$ using intramuscular iron administration but not in the two trials ${ }^{23} 32$ that used oral administration. Nausea was reported in four trials ${ }^{18283133}$; two trials $^{28} 31$ using intravenous administration of iron reported significantly increased nausea, whereas nausea was not increased in patients who received iron by oral administration. ${ }^{183}$ Constipation was reported in one trial ${ }^{18}$ and diarrhoea in two trials ${ }^{1831}$ (see online supplementary appendix
10). Adherence with the study intervention was reported in 13 trials. $^{18} 19$ 22-29 323335 Iron supplementation was not associated with differential rates of medication adherence (RR 1.0; CI 95\% 0.99 to 1.01; I $\mathrm{I}^{2} 0 \%$; 12 trials; 958 participants). The route of administration of the study intervention was also not associated with differences in adherence (see online supplementary appendix 11).

\section{DISCUSSION}

In iron-deficient but non-anaemic adults, we found iron supplementation was associated with reduced subjective measures of fatigue but had no significant impact on objective physical capacity. Given iron deficiency is the most prevalent micronutrient deficiency worldwide, ${ }^{2}$ there is a discrepant lack of robust evidence evaluating iron supplementation in the absence of anaemia across important patient populations. Despite rigorous and systematic methodology, we were only able to identify 18

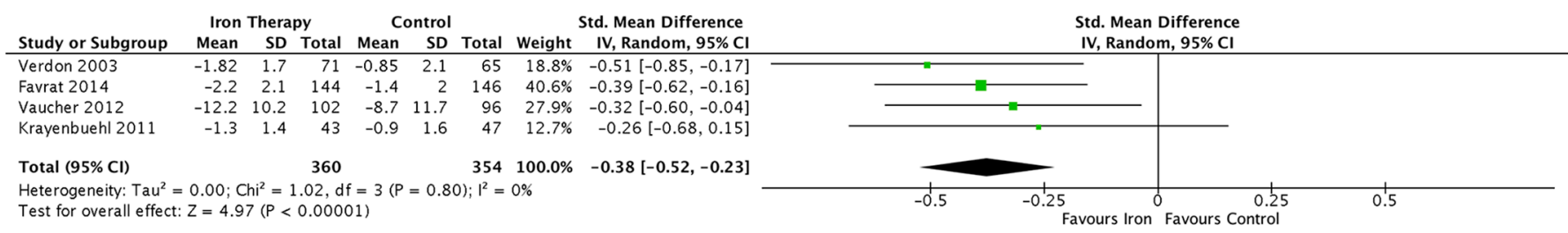

Figure 2 The effect of iron supplementation on patient-reported fatigue using validated fatigue scores. Iron supplementation was associated with a reduction in subjective measures of fatigue when assessed by either the Piper Fatigue Scale, the Current and Past Psychological State Scale, visual analogue scale or Brief Fatigue Inventory questionnaire. 


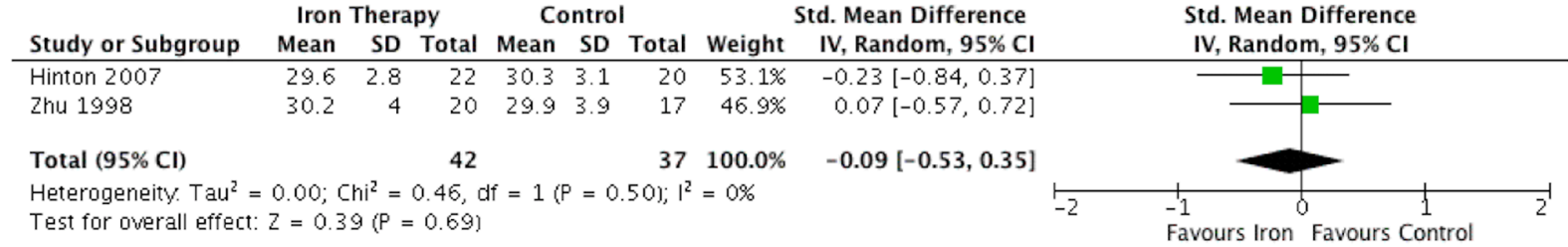

\section{$3 A .15 \mathrm{~km}$ time trial}

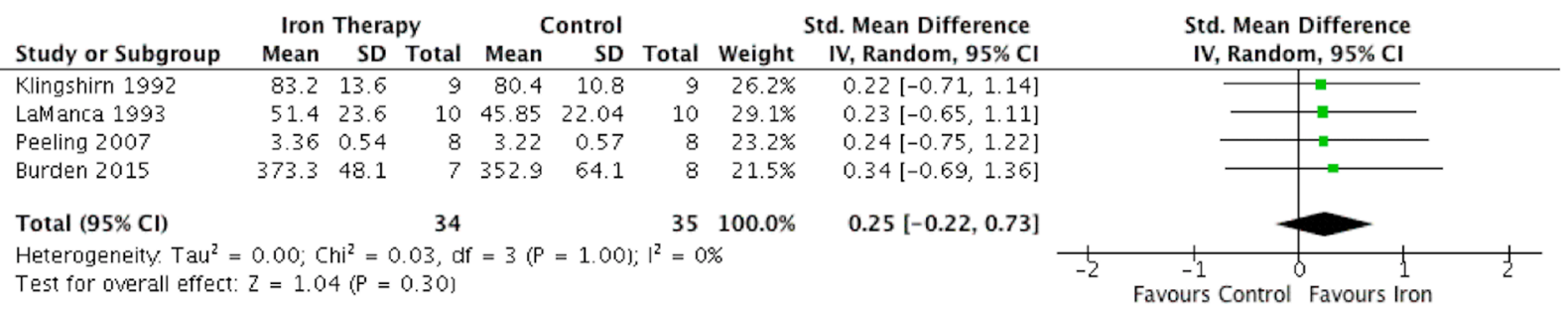

\section{B. Time to exhaustion}

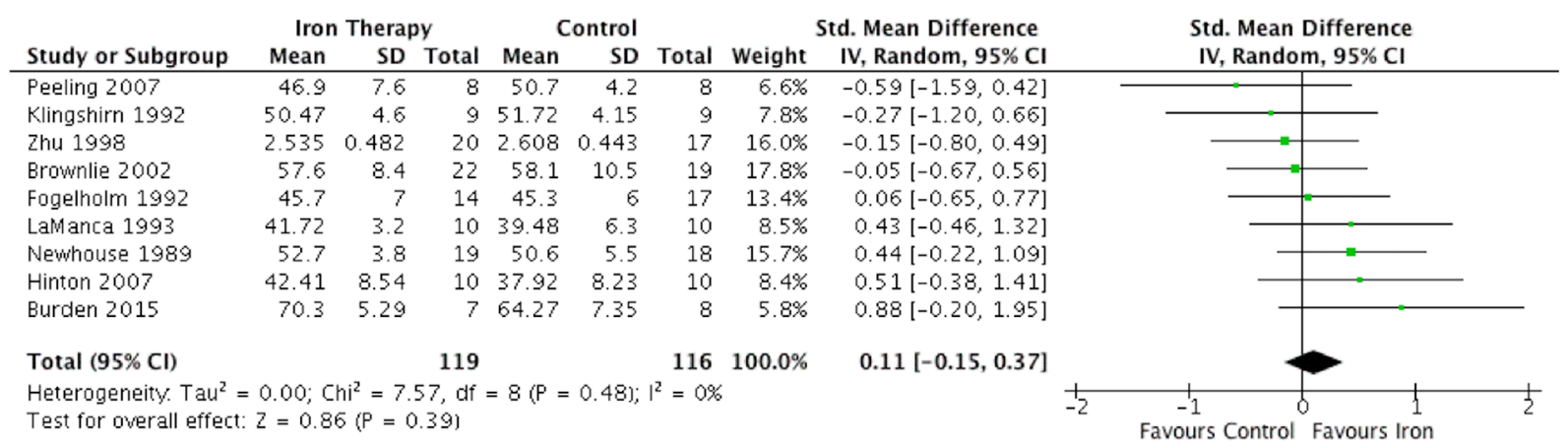

\section{C. Oxygen consumption $\left(\mathrm{VO}_{2}\right.$ max)}

Figure 3 The effect of iron supplementation on measures of physical capacity. Iron supplementation was not associated with a reduction in objective measures of physical capacity when assessed by either maximal oxygen consumption and timed methods of exercise testing.

trials enrolling 1170 adults, representing a minute fraction of affected individuals.

While treatment of iron deficiency in the absence of anaemia is associated with reduced subjective fatigue, whether this translates to clinically meaningful outcomes, including quality of life, work absenteeism, job or athletic performance is uncertain. Contrary to iron deficiency with established anaemia, lack of robust data in iron-deficient but non-anaemic individuals is reflected in the under-representation of guideline recommendations pertaining to this larger population. The proportion of iron-deficient, non-anaemic individuals who receive supplementation is further unknown.

Our systemic review builds on the results of three published evidence syntheses evaluating iron supplementation. ${ }^{37-39}$ In a systematic review of healthy menstruating women, iron supplementation, irrespective of iron status or anaemia, improved haemoglobin and measures of iron stores. ${ }^{37}$ Two systematic reviews included studies of pregnant women, blood donors and children and included data from both randomised and non-randomised trials. ${ }^{38} 39$ These studies concluded benefit of iron supplementation, although in the review by Yokoi and $\mathrm{Konomi}^{39}$, the benefit was limited to randomised controlled trials. Despite the high prevalence of iron deficiency, significant heterogeneity in patient populations and study designs and absence of data pertaining to objective muscle performance limits the generalisability of these findings.

In trials where a proportion of participants were anaemic at enrolment and with the knowledge that anaemia results in decreased physical capacity, iron supplementation has previously been associated with improved maximal and submaximal exercise performance. ${ }^{5-8}$ We found insufficient evidence to suggest that iron supplementation improves exercise capacity in iron-depleted non-anaemic adults, differing from the results of physiological experiments that describe $\mathrm{VO}_{2}$ max improvements with iron 
supplementation, independent of haemoglobin. ${ }^{40}$ These findings were postulated to be secondary to iron-mediated improvements in muscle oxidative capacity and improved mitochondrial function, the validity of which is unclear. ${ }^{40}$

A potential weakness our systematic review is the difficulty masking oral iron due to predictable gastrointestinal side effects and changes in stool colour and the impact of imperfect blinding on subjective measures of fatigue. However, despite this, fatigue was consistently reduced in trials evaluating both oral $(\mathrm{n}=2)$ and intravenous $(n=2)$ iron preparations. Healthy women comprised the study population in 15 of 18 included trials; subjective measures of fatigue may not consistently apply to other at-risk populations. The duration of follow-up was relatively short (57 days; range 28-112 days) and perhaps too brief to expect significant changes in muscle metabolism or function. Finally, the lack of systematic reporting of adverse events impairs our ability to draw conclusions regarding the incidence of these events and tolerability of iron therapy.

The strengths of this review include the comprehensiveness of the search strategy, which included electronic databases, trial registries and forward searches. We used an a priori published protocol and followed established methodological guidelines concerning the conduct and reporting of this review. We synthesised patient-centred outcomes and evaluated efficacy in the context of relevant safety outcomes and adverse events. In contrast to the systematic review of Low et al, ${ }^{37}$ we excluded studies that enrolled patients with anaemia at baseline. ${ }^{37}$ While cut-offs for anaemia varied slightly among included trials, this important inclusion criteria reduces (but may not eliminate) the probability that changes in fatigue or muscle function are due to correction of anaemia. While the duration of follow-up in most studies was modest, the mean daily elemental iron dose $(86.9 \pm 49.1 \mathrm{mg})$ reflects a recommended 'treatment' for patients with iron-deficiency anaemia. ${ }^{41}$

In IDNA adults, iron supplementation is associated with reduced subjective measures of fatigue but not with objective improvements in physical capacity. Given the global prevalence of both iron deficiency and fatigue, patients and practitioners could consider consumption of ironrich foods or iron supplementation to improve symptoms of fatigue in the absence of documented anaemia.

\footnotetext{
Author affiliations

${ }^{1}$ Department of Internal Medicine, Section of Medical Oncology and Haematology, University of Manitoba, Winnipeg, Manitoba, Canada

${ }^{2}$ Department of Medical Oncology and Haematology, CancerCare Manitoba, Winnipeg, Manitoba, Canada

${ }^{3}$ Applied Health Sciences, Faculty of Kinesiology and Recreation Management, University of Manitoba, Winnipeg, Manitoba, Canada

${ }^{4}$ Department of Internal Medicine, Faculty of Medicine, University of Manitoba, Winnipeg, Manitoba, Canada

${ }^{5}$ George \& Fay Yee Centre for Healthcare Innovation, University of Manitoba/ Winnipeg Regional Health Authority, Winnipeg, Manitoba, Canada

${ }^{6}$ Department of Community Health Sciences, University of Manitoba, Winnipeg, Manitoba, Canada
}

${ }^{7}$ Department of Internal Medicine, Section of Gastroenterology, University of Manitoba, Winnipeg, Manitoba, Canada

${ }^{8}$ Population Health and Optimal Health Practices Research Unit, TraumaEmergency-Critical Care Medicine, CHU de Québec-Université Laval Research Center, Québec City, Québec, Canada

${ }^{9}$ Department of Anesthesiology and Critical Care Medicine, Division of Critical Care Medicine, Faculty of Medicine, Université Laval, Québec City, Québec, Canada

${ }^{10}$ Clinical Epidemiology Program, Ottawa Hospital Research Institute (OHRI);

Department of Medicine, University of Ottawa, Ottawa, Ontario, Canada

Contributors All authors meet ICMJE criteria for authorship. Two researchers (BH and $\mathrm{DH}$ ) lead and coordinated all aspects of the review, including but not limited to preparation of the literature search, screening relevant material, data analysis and extraction, interpretation of the results of the meta-analytic procedures, bias investigation and preparation of the final report; three second reviewers (JG, ER, BP) conducted independent screening of relevant material, extracted and analysed data and aided in report preparation; one haematologist/ intensivist (RZ), methodologist (DAF) and anaesthetist/intensivist (AFT) with expertise in systematic reviews provided content expertise and methodological input and resolved disagreement among reviewers; one systematic review expert (AMAS) provided methodological input; two haematologists (DSH and ER), one gastroenterologist (CNB) and one statistician (RR) provided content expertise. All authors were involved in the process of study design and manuscript review.

Funding This research received no specific grant from any funding agency in the public, commercial or not-for-profit sectors.

Competing interests None declared.

Patient consent Not required.

Provenance and peer review Not commissioned; externally peer reviewed.

Data sharing statement We are submitting (in our manuscript and supplementary files) all planned data analyses.

Open Access This is an Open Access article distributed in accordance with the Creative Commons Attribution Non Commercial (CC BY-NC 4.0) license, which permits others to distribute, remix, adapt, build upon this work non-commercially, and license their derivative works on different terms, provided the original work is properly cited and the use is non-commercial. See: http://creativecommons.org/ licenses/by-nc/4.0/

(c) Article author(s) (or their employer(s) unless otherwise stated in the text of the article) 2018. All rights reserved. No commercial use is permitted unless otherwise expressly granted.

\section{REFERENCES}

1. Kassebaum NJ, Jasrasaria R, Naghavi M, et al. A systematic analysis of global anemia burden from 1990 to 2010. Blood 2014;123:615-24.

2. McLean E, Cogswell M, Egli I, et al. Worldwide prevalence of anaemia, WHO Vitamin and Mineral Nutrition Information System, 1993-2005. Public Health Nutr 2009;12:444-54.

3. Milman N. Anemia: still a major health problem in many parts of the world!. Ann Hematol 2011;90:369-77.

4. Assessing the iron status of populations: including literature reviews: report of a Joint World Health Organization/Centers for Disease Control and Prevention Technical Consultation on the Assessment of Iron Status on the Population Level. Geneva, Switzerland, 2004:108.

5. Dallman PR. Iron deficiency: does it matter? J Intern Med 1989;226:367-72.

6. Eichner ER. Fatigue of anemia. Nutr Rev 2001;59:S17-S19.

7. Florencio CA. Effects of iron and ascorbic acid supplementation on hemoglobin level and work efficiency of anemic women. J Occup Med 1981;23:699-704.

8. Haas JD, Brownlie T. Iron deficiency and reduced work capacity: a critical review of the research to determine a causal relationship. $J$ Nutr 2001;131:676S-90.

9. Lukaski HC. Vitamin and mineral status: effects on physical performance. Nutrition 2004;20:632-44.

10. Lewis $\mathrm{G}$, Wessely $\mathrm{S}$. The epidemiology of fatigue: more questions than answers. J Epidemiol Community Health 1992;46:92-7.

11. Jason LA, Evans M, Brown M, et al. What is fatigue? Pathological and nonpathological fatigue. Pm R 2010;2:327-31.

12. Hurrie D, Graham J, Rimmer E, et al. Efficacy of iron replacement therapy on fatigue and work capacity in non-anemic adults with iron depletion: a systematic review of randomized controlled trials. 
PROSPERO International prospective register of systematic reviews, 2014.

13. The Cochrane Collaboration. Cochrane handbook for systematic reviews of interventions, 2009.

14. Liberati A, Altman DG, Tetzlaff J, et al. The PRISMA statement for reporting systematic reviews and meta-analyses of studies that evaluate healthcare interventions: explanation and elaboration. BMJ 2009;339:b2700.

15. Higgins JP, Thompson SG. Quantifying heterogeneity in a metaanalysis. Stat Med 2002;21:1539-58.

16. Sterne JA, Egger M, Smith GD. Systematic reviews in health care: investigating and dealing with publication and other biases in metaanalysis. BMJ 2001;323:101-5

17. Hinton PS, Giordano C, Brownlie T, et al. Iron supplementation improves endurance after training in iron-depleted, nonanemic women. J Appl Physiol 2000;88:1103-11.

18. Leonard AJ, Chalmers KA, Collins CE, et al. Comparison of two doses of elemental iron in the treatment of latent iron deficiency: efficacy, side effects and blinding capabilities. Nutrients 2014;6:1394-405.

19. Brownlie T, Utermohlen V, Hinton PS, et al. Marginal iron deficiency without anemia impairs aerobic adaptation among previously untrained women. Am J Clin Nutr 2002;75:734-42.

20. Brutsaert TD, Hernandez-Cordero S, Rivera J, et al. Iron supplementation improves progressive fatigue resistance during dynamic knee extensor exercise in iron-depleted, nonanemic women. Am J Clin Nutr 2003;77:441-8.

21. Donangelo CM, Woodhouse LR, King SM, et al. Supplemental zinc lowers measures of iron status in young women with low iron reserves. J Nutr 2002;132:1860-4.

22. Hinton PS, Sinclair LM. Iron supplementation maintains ventilatory threshold and improves energetic efficiency in iron-deficient nonanemic athletes. Eur J Clin Nutr 2007;61:30-9.

23. Klingshirn LA, Pate RR, Bourque SP, et al. Effect of iron supplementation on endurance capacity in iron-depleted female runners. Med Sci Sports Exerc 1992;24:819-24.

24. Newhouse IJ, Clement DB, Taunton JE, et al. The effects of prelatent/ latent iron deficiency on physical work capacity. Med Sci Sports Exerc 1989;21:263-8.

25. Zhu YI, Haas JD. Altered metabolic response of iron-depleted nonanemic women during a 15-km time trial. J Appl Physiol 1998;84:1768-75.

26. LaManca JJ, Haymes EM. Effects of iron repletion on VO2max, endurance, and blood lactate in women. Med Sci Sports Exerc 1993;25:1386-92.
27. Burden RJ, Pollock N, Whyte GP, et al. Effect of intravenous iron on aerobic capacity and iron metabolism in elite athletes. Med Sci Sports Exerc 2015;47:1399-407.

28. Favrat B, Balck K, Breymann C, et al. Evaluation of a single dose of ferric carboxymaltose in fatigued, iron-deficient women--PREFER a randomized, placebo-controlled study. PLoS One 2014;9:e94217.

29. Flink $\mathrm{H}$, Tegelberg $\mathrm{A}$, Thörn $\mathrm{M}$, et al. Effect of oral iron supplementation on unstimulated salivary flow rate: a randomized, double-blind, placebo-controlled trial. J Oral Pathol Med 2006;35:540-7.

30. Fogelholm M, Jaakkola L, Lampisjärvi T. Effects of iron supplementation in female athletes with low serum ferritin concentration. Int J Sports Med 1992;13:158-62.

31. Krayenbuehl PA, Battegay E, Breymann C, et al. Intravenous iron for the treatment of fatigue in nonanemic, premenopausal women with low serum ferritin concentration. Blood 2011;118:3222-7.

32. Vaucher P, Druais PL, Waldvogel S, et al. Effect of iron supplementation on fatigue in nonanemic menstruating women with low ferritin: a randomized controlled trial. CMAJ 2012;184:1247-54.

33. Verdon F, Burnand B, Stubi CL, et al. Iron supplementation for unexplained fatigue in non-anaemic women: double blind randomised placebo controlled trial. BMJ 2003;326:1124.

34. Peeling P, Blee T, Goodman C, et al. Effect of iron injections on aerobic-exercise performance of iron-depleted female athletes. Int $J$ Sport Nutr Exerc Metab 2007;17:221-31.

35. Moafi A, Rahgozar S, Hanian M, et al. The effects of supplemental iron on educational achievements of students with "iron deficiency without anemia": a randomized, double-blind, placebo-controlled trial. HealthMED 2012;6:2047-51.

36. Leonard AJ, Chalmers KA, Collins CE, et al. A study of the effects of latent iron deficiency on measures of cognition: a pilot randomised controlled trial of iron supplementation in young women. Nutrients 2014;6:2419-35.

37. Low MS, Speedy J, Styles CE, et al. Daily iron supplementation for improving anaemia, iron status and health in menstruating women. Cochrane Database Syst Rev 2016;4:CD009747.

38. Pratt JJ, Khan KS. Non-anaemic iron deficiency-a disease looking for recognition of diagnosis: a systematic review. Eur J Haematol 2016;96:618-28

39. Yokoi K, Konomi A. Iron deficiency without anaemia is a potential cause of fatigue: meta-analyses of randomised controlled trials and cross-sectional studies. Br J Nutr 2017;117:1422-31.

40. Friedmann B, Weller E, Mairbaurl $\mathrm{H}$, et al. Effects of iron repletion on blood volume and performance capacity in young athletes. Med Sci Sports Exerc 2001;33:741-6.

41. Camaschella C. Iron-deficiency anemia. N Engl J Med 2015;372:1832-43. 